\title{
Características de pacientes atendidos en dos clínicas de anticoagulación de Colombia
}

\author{
Characteristics of patients in two anticoagulation clinics in Colombia
}

\author{
Ana Montenegro ${ }^{1,2}$, Maribel Plaza $^{3}$, Rafael Álvarez ${ }^{2,4}$, Angie Pinto ${ }^{5}$, Luis Gómez ${ }^{6}$, Jhon Castaño7, \\ Diana Giraldo y Andrés Buitrago ${ }^{2,8}$
}

${ }^{1}$ Clínica de Anticoagulación, Clínica de Enfermedades Vasculares, Fundación Santa Fe de Bogotá, Bogotá; ${ }^{2}$ Facultad de Medicina, Universidad de los Andes, Bogotá; ${ }^{3}$ Servicio de Medicina Interna y Medicina Vascular, Universidad de Antioquia, Medellín; ${ }^{4}$ Clínica de Enfermedades Vasculares, Fundación Santa Fe de Bogotá, Bogotá; ${ }^{5}$ Facultad de Medicina, Universidad de Antioquia, Medellín; ${ }^{6}$ Clínica Medellín, Universidad de Antioquia, Medellín; ${ }^{7}$ Hospital San Vicente Fundación, Universidad de Antioquia, Medellín; ${ }^{8}$ Servicio de Cardiología, Fundación Santa Fe de Bogotá, Bogotá. Colombia

\section{Resumen}

Objetivo: Establecer las características demográficas, clínicas y terapéuticas de los pacientes anticoagulados atendidos en las clínicas de anticoagulación del Hospital Universitario Fundación Santa Fe de Bogotá (HUFSFB) y del Hospital Universitario San Vicente Fundación Medellín (HUSVF), en los años 2015 y 2016. Método: Se realizó un estudio descriptivo, retrospectivo y transversal. La población de referencia la conformaron los pacientes atendidos en las clínicas de anticoagulación del HUSVF y el HUFSFB en el periodo comprendido entre marzo de 2015 y marzo de 2016. La información se tomó de los registros clínicos de ambos hospitales y para el análisis se utilizó estadística descriptiva. Resultados: Se evaluaron 502 pacientes en las dos clínicas de anticoagulación, con una edad promedio de 64 años en el HUFSFB y de 61 años en el HUSVF. La principal indicación de anticoagulación fue fibrilación o flutter auricular en el HUSVF (46.5\%) y enfermedad tromboembólica venosa en el HUFSFB (69.7\%). El 100\% de los pacientes del HUSVF fueron anticoagulados con warfarina, frente a solo el 23\% de los del HUFSFB; el tiempo en rango terapéutico fue del 69\% en el HUFSFB y del 55\% en el HSVF. Se presentó retrombosis en tres pacientes en cada una de las instituciones, y los eventos de sangrado fueron 7 (3,8\%) en el HUFSFB y 30 (9,3\%) en el HUSVF. Conclusiones: Las diferencias administrativas entre las dos instituciones se ven reflejadas en los resultados obtenidos. Existe, ligada al sistema de salud de nuestro país, una gran diferencia en la población, las enfermedades y el acceso a los servicios y a los medicamentos.

Palabras clave: Anticoagulación. Warfarina. Anticoagulantes orales de acción directa.

\section{Abstract}

Objective: To establish the demographic, clinical and therapeutic characteristics of the anticoagulated patients treated at the anticoagulation clinics of Fundación Santa Fe de Bogotá (HUSVF) and Hospital Universitario San Vicente Fundación MedeIlin (HUFSFB), between 2015 and 2016. Method: A transversal retrospective descriptive study was carried out. The reference population consist in patients treated in the anticoagulation clinics of the HUSVF in Medellin and the HUFSFB in Bogotá between March 2015 to 2016. The information was taken from the clinical records of both hospitals and descriptive statistics were used for the data analysis. Results: A total of 502 patients were evaluated in both anticoagulation clinics. Patients'

\section{Correspondencia:}

*Rafael Álvarez

E-mail: Rd.alvarez867@uniandes.edu.co
Fecha de recepción: 19-10-2018

Fecha de aceptación: 25-03-2021

DOI: 10.24875/RCCAR.M21000065
Disponible en internet: 18-10-2021 Rev Colomb Cardiol. 2021;28(4):353-359 www.rccardiologia.com 0120-5633 / C 2021 Sociedad Colombiana de Cardiología y Cirugía Cardiovascular. Publicado por Permanyer. Este es un artículo open access bajo la licencia CC BY-NC-ND (http://creativecommons.org/licenses/by-nc-nd/4.0/). 
average age in HUFSFB was 64 years while in HUSVF was 61; the main pathology that required anticoagulation was the fibrillation/auricular flutter in HUSVF (46,5\%) and venous thromboembolic disease in HUFSFB (69.7\%). 100\% of HUSVF patients are anticoagulated using warfarin and only $23 \%$ of those in HUFSFB; the time in therapeutic range (TTR) was $69 \%$ in HUFSFB and 55\% in HSVF. Re-thrombosis was presented in 3 patients in each of the institutions and the bleeding events were 7 (3.8\%) in HUFSFB and 30 (9.3\%) in HUSVF. Conclusions: The administrative differences between the two institutions are reflected in the results obtained; there is a great difference in the population, pathologies, access to services and medicines, linked to the health system of our country.

Key words: Anticoagulation. Warfarin. Direct acting oral anticoagulants.

\section{Introducción}

La enfermedad tromboembólica venosa y la fibrilación auricular representan una alta carga de enfermedad para la sociedad actual ${ }^{1}$. Los anticoagulantes son efectivos para reducir la recurrencia de la enfermedad tromboembólica venosa, los eventos embólicos sistémicos asociados a fibrilación auricular y otras complicaciones $^{2,3}$. Por más de 60 años, la heparina y la warfarina han sido la piedra angular de la anticoagulación; sin embargo, tienen amplias limitaciones, como la necesidad de una cuidadosa monitorización o control con ajuste de dosis, múltiples interacciones medicamentosas y un estrecho rango terapéutico, entre otras $^{4,5}$. Los anticoagulantes de acción directa (ACOD) han surgido como alternativa terapéutica, contribuyendo a disminuir los efectos adversos y las complicaciones, como el sangrado intracraneal, en pacientes que necesiten estar anticoagulados ${ }^{6,7}$.

Como respuesta a la necesidad de mantener una monitorización más estrecha a los pacientes anticoagulados, y con miras a garantizar su seguridad, se han creado las clínicas de anticoagulación, con las cuales algunos estudios han demostrado disminuir la incidencia de complicaciones ${ }^{8}$. En el caso de la warfarina, los pacientes controlados en las clínicas de anticoagulación aumentan el tiempo en rango terapéutico (TRT) con una reducción de los desenlaces adversos ${ }^{9,10}$. En Colombia existe un creciente número de clínicas de anticoagulación en aras de garantizar unas mayores seguridad y eficacia terapéutica a los pacientes; sin embargo, en nuestro país hay carencia de estudios prospectivos y retrospectivos en los cuales se caracterice la población de pacientes anticoagulados, sus desenlaces y sus efectos adversos. Sin dicha caracterización, es difícil realizar intervenciones efectivas a poblaciones específicas y obtener resultados cuantificados.

El Hospital Universitario Fundación Santa Fe de Bogotá (HUFSFB) y el Hospital Universitario San Vicente Fundación (HUSVF) de Medellín han encaminado esfuerzos con el fin de mejorar la evidencia disponible sobre los pacientes anticoagulados que asisten a sus respectivas clínicas de anticoagulación. En el HUSVF, en 2002, Ocampo, et al..$^{11}$ realizaron un estudio con el fin de describir las características de los pacientes inscritos en el programa de anticoagulación, en el que fueron analizados los datos de 139 pacientes, todos anticoagulados con warfarina, y se encontró un TRT del $41 \%$ con un promedio de 4.5 controles al año. De la misma manera, en 2013, Taboada, et al. ${ }^{12}$, en la clínica de anticoagulación del HUFSFB, incluyeron 159 pacientes, todos anticoagulados con warfarina y con un TRT del 53\%; hallazgo similar al de la nueva evaluación realizada por Laverde, et al. ${ }^{13}$ en el año 2015 para aquellos manejados con warfarina.

Pese a todos estos esfuerzos, se hace necesario establecer las características demográficas, clínicas y terapéuticas de la población de pacientes pertenecientes a una clínica de anticoagulación de ambos hospitales universitarios con el fin de obtener nuevos datos sobre indicaciones, desenlaces, efectos adversos y efectividad terapéutica, para dar continuidad con los estudios realizados anteriormente y contrastar los resultados obtenidos en ambas instituciones. También hace falta conocer el papel de los ACOD en el tratamiento de los pacientes, sus desenlaces y efectos adversos, y realizar comparaciones con la terapia tradicional.

El propósito de este estudio fue determinar las características demográficas, clínicas y terapéuticas de los pacientes anticoagulados atendidos en las clínicas de anticoagulación de estas dos instituciones, con el propósito de sentar unas bases para ser referente en el manejo de pacientes en terapia anticoagulante en el país.

\section{Método}

Se realizó un estudio descriptivo, retrospectivo y transversal. La población de referencia la conformaron los pacientes atendidos en las clínicas de anticoagulación del HUSVF en Medellín y del HUFSFB en Bogotá, en el 
periodo comprendido entre el 1 de marzo de 2015 y el 1 de marzo de 2016. Como se estudió toda la población, no fue necesario calcular el tamaño de muestra.

Se incluyeron pacientes de todas las edades y de ambos sexos, pertenecientes a la clínica de anticoagulación de cada una de las instituciones, en tratamiento anticoagulante por cualquier patología con indicación clara, que tuvieran al menos cuatro consultas de seguimiento en la clínica en el último año, con al menos cuatro mediciones del INR (International Normalized Ratio) en caso de que recibieran como anticoagulante warfarina, registrados en la tarjeta de seguimiento (HUSVF), en la historia clínica de control o en el reporte del laboratorio clínico (HUFSFB). Se excluyeron los pacientes que cambiaron de terapia por algún motivo o que no completaron el esquema de tratamiento; en el HUFSFB no se incluyeron registros de INR del laboratorio clínico que correspondieran a pacientes hospitalizados.

Las variables consideradas fueron demográficas, clínicas y terapéuticas a partir de los registros de cada una de las clínicas y de la historia clínica electrónica. Se utilizó como instrumento de recolección de la información la herramienta Excel de Microsoft, y para el análisis de los datos se realizó estadística descriptiva con medidas de tendencia central y distribuciones. Para calcular el TRT se utilizó el método de interpolación de Rosendaal.

Para la realización del estudio se tuvieron en cuenta los aspectos éticos que rigen este tipo de investigaciones y se contó con el permiso institucional.

\section{Resultados}

Entre las dos instituciones se incluyeron 502 pacientes: 182 en el HUFSFB y 320 en el HUSVF. De la población de pacientes, el $55.1 \%$ eran de sexo femenino; con una distribución similar entre las dos instituciones (Tabla 1). La edad promedio de los pacientes también fue similar: 64 años en el HUFSFB y 61 años en el HUSVF (Tabla 2). Con respecto a la seguridad social, se encontró una diferencia clara, ya que el $86.81 \%$ de los pacientes en el HUFSFB son de medicina privada o particulares, mientras que en el HUSVF la mayor población pertenece al régimen subsidiado (51.2\%).

\section{Tipo de anticoagulación y seguimiento}

En el HUSVF, todos los pacientes fueron manejados con warfarina independientemente de su enfermedad. En el HUFSFB, la principal terapia anticoagulante indicada
Tabla 1. Distribución de los pacientes por institución según el sexo

\begin{tabular}{|l|c|c|c|c|c|c|}
\hline $\begin{array}{l}\text { Variable } \\
\text { Sexo }\end{array}$ & HUFSFB & $\%$ & HUSVF & $\%$ & Total & $\%$ \\
\hline Femenino & 92 & 50.55 & 185 & 57.81 & 277 & 55.18 \\
\hline Masculino & 90 & 49.45 & 135 & 42.19 & 226 & 44.82 \\
\hline
\end{tabular}

HUFSFB: Hospital universitario Fundación Santa Fe de Bogotá; HUSVF: Hospital universitario San Vicente Fundación de Medellín.

Tabla 2. Distribución de los pacientes por institución según los intervalos de edad

\begin{tabular}{|l|c|c|c|c|}
\hline $\begin{array}{l}\text { Variable } \\
\text { Edad (años) }\end{array}$ & HUFSFB & $\%$ & HSVF & $\%$ \\
\hline $18-30$ & 7 & 3.85 & 24 & 7.50 \\
\hline $31-45$ & 26 & 14.29 & 35 & 10.94 \\
\hline $46-60$ & 33 & 18.13 & 83 & 32.19 \\
\hline $61-75$ & 62 & 34.07 & 103 & 23.44 \\
\hline$>75$ & 54 & 29.67 & 75 & \\
\hline
\end{tabular}

HUFSFB: Hospital universitario Fundación Santa Fe de Bogotá; HSVF: Hospital universitario Fundación Santa Fe de Bogotá.

fueron los ACOD (64.84\%), siendo el rivaroxabán el más usado (31.87\%), seguido por el apixabán $(25.27 \%$ ) y con menor frecuencia el dabigatrán (7.69\%). Por el contrario, la warfarina se usó tan solo en el $23.08 \%$ de la población.

El TRT promedio calculado por el método de Rosendaal fue del $55.06 \%$ en el HUSVF y del $69.6 \%$ en la población anticoagulada con warfarina atendida en el HUFSFB. El seguimiento promedio fue mayor en el HUSVF (20 meses) que en el HUFSFB (19 meses aproximadamente), con un número de visitas promedio en el año similar en las dos instituciones (5.3 en el $\mathrm{HU}$ FSFB y 6.5 en el HUSVF).

\section{Indicación de la anticoagulación y factores desencadenantes de enfermedad tromboembólica venosa}

La indicación de anticoagulación más frecuente en el HUFSFB fueron los eventos tromboembólicos venosos, mientras que en el HUSVF fueron la fibrilación y el flutter auricular (Fig. 1).

Entre los factores desencadenantes de enfermedad tromboembólica venosa, en el HUSVF un $58.7 \%$ correspondieron a eventos no provocados y el principal factor desencadenante fue el síndrome antifosfolípido. 


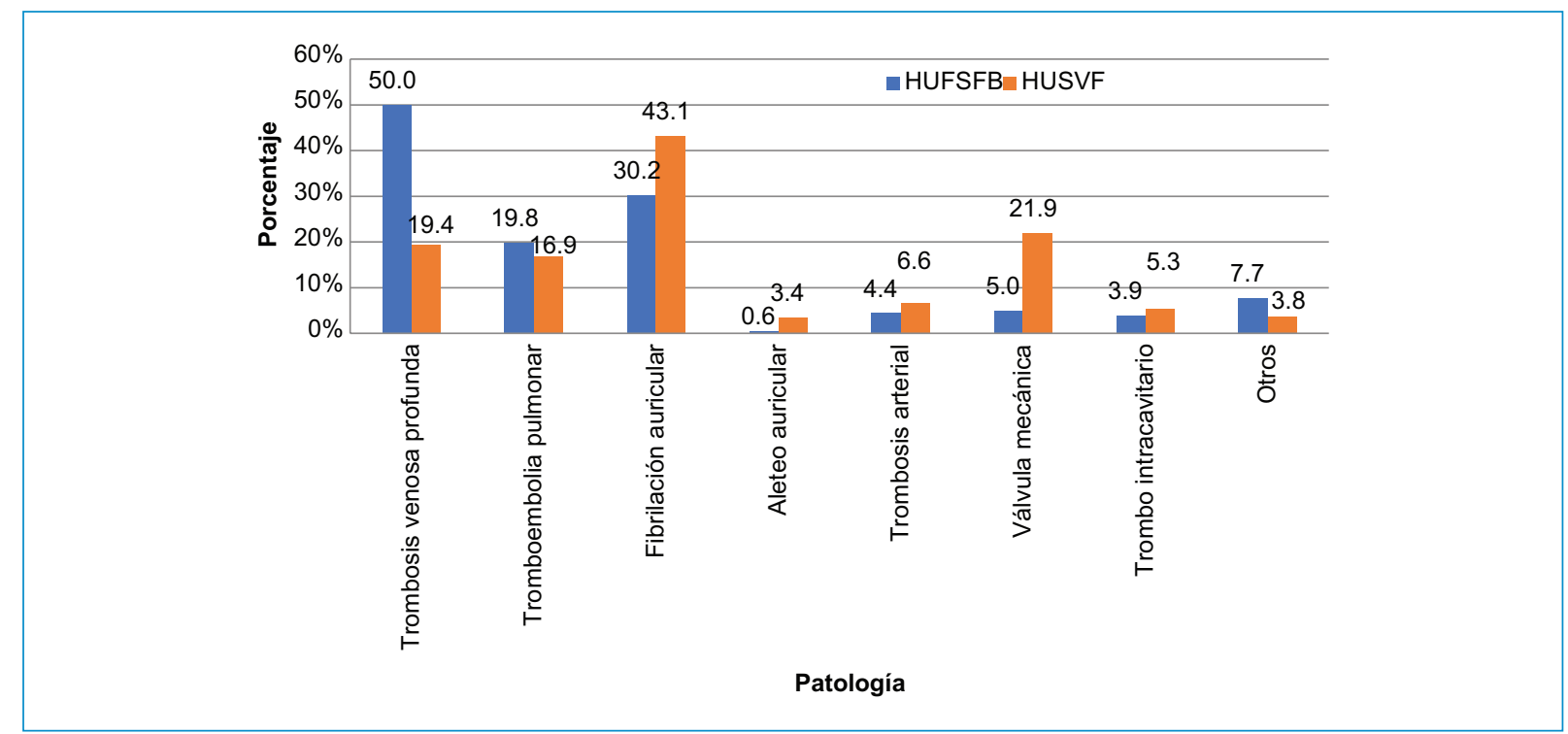

Figura 1. Distribución de los pacientes según institución e indicación de la anticoagulación.

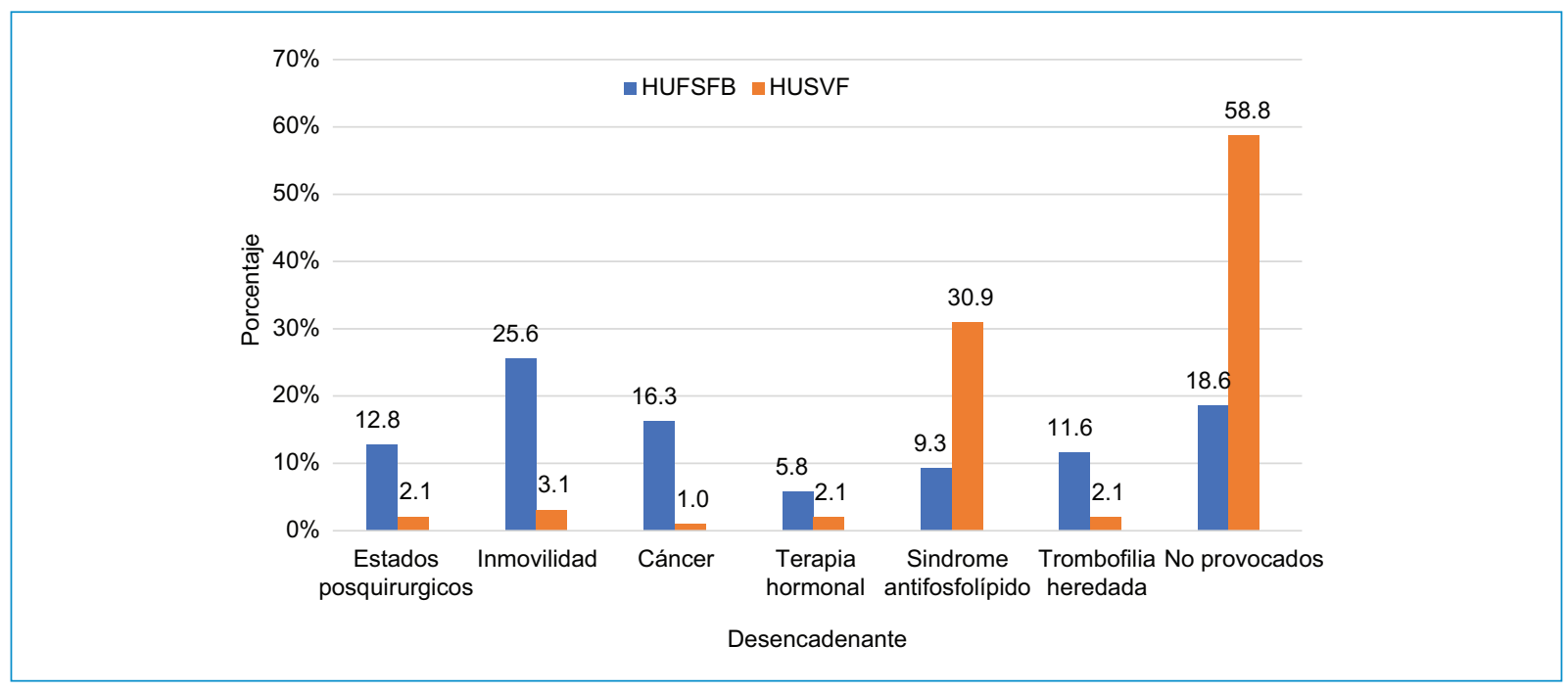

Figura 2. Distribución de los pacientes según institución y factor desencadenante de la enfermedad tromboembólica venosa.

En el HUFSFB, la inmovilidad fue desencadenante en un $25.5 \%$ y el cáncer en un $16.2 \%$, y los eventos no provocados correspondieron al $18.6 \%$ (Fig. 2).

\section{Complicaciones asociadas: retrombosis y sangrado}

El porcentaje de sangrados en el HUFSFB fue del $3.83 \%$, de los cuales el $0.55 \%$ fueron sangrados mayores y el $3.28 \%$ fueron sangrados menores. En el
HUSVF, el sangrado ocurrió en el $9.38 \%$ de los pacientes, principalmente sangrados menores (7.5\%) (Tabla 3). El porcentaje de retrombosis en el HUFSFB fue del $1.64 \%$ y en el HUSVF fue del $0.94 \%$ (Tabla 3 ).

\section{Discusión}

El HUFSFB y el HUSVF son entidades administrativamente diferentes. Aunque ambas son de carácter privado, existen diferencias en las poblaciones que 
Tabla 3. Distribución de los pacientes por institución según el sangrado y la retrombosis

\begin{tabular}{|l|c|c|c|c|}
\hline Variable & HUFSFB & $\%$ & HUSVF & $\%$ \\
\hline Retrombosis & 3 & $1.64 \%$ & 3 & $0.94 \%$ \\
\hline Sangrado mayor & 1 & $0.55 \%$ & 6 & $1.88 \%$ \\
\hline Sangrado menor & 6 & $3.28 \%$ & 24 & $7.50 \%$ \\
\hline Sangrado total & 7 & $3.83 \%$ & 30 & $9.38 \%$ \\
\hline
\end{tabular}

HUFSFB: Hospital universitario Fundación Santa Fe de Bogotá; HUSVF: Hospital universitario San Vicente Fundación de Medellín.

asisten. En el HUSVF, en su gran mayoría son pacientes pertenecientes al régimen subsidiado, mientras que en el HUFSFB la mayor parte de los pacientes pertenecen a medicina prepagada o de pago directo, lo cual tiene una implicación importante en las grandes diferencias entre las clínicas de anticoagulación de las dos instituciones.

En lo que respecta a la población en términos de sexo, el predominante es el femenino en las dos clínicas, lo cual es acorde con la mayor prevalencia de enfermedad tromboembólica en las mujeres ${ }^{14,15}$. La edad también fue similar entre las dos instituciones, en promedio mayor de 60 años, lo cual también está acorde con los reportes de la literatura, en especial para la fibrilación auricular, la cual incrementa su incidencia de forma proporcional con la edad ${ }^{16,17}$.

Con respecto al tratamiento utilizado, en el HUSVF todos los pacientes se encontraban con warfarina, a diferencia del HUFSFB, en donde la mayoría recibían ACOD. Cabe destacar que este escenario ha cambiado drásticamente y se hace necesario realizar un seguimiento a los programas de anticoagulación para poder comprender el impacto en los desenlaces de los pacientes. Laverde, et al. ${ }^{13}$ reportaron que en la clínica de anticoagulación del HUFSFB, entre los años 2008 y 2013 , el $71 \%$ de los pacientes se encontraban utilizando warfarina y tan solo el $32 \%$ recibían ACOD. En nuestro estudio se encontró que el $64 \%$ de la población de esa institución está anticoagulada con ACOD. Esta tendencia es un reflejo de lo que ocurre en todo el mundo con la terapia anticoagulante en enfermedades como la fibrilación auricular y la enfermedad tromboembólica venosa ${ }^{18,19}$. Los registros de la cohorte GARFIELD para fibrilación auricular han demostrado que existe un cambio sustancial en el patrón de uso de la terapia anticoagulante desde la aparición de los ACOD, con un incremento del $70 \%$ en el uso de terapia antitrombótica, dado principalmente por el mayor uso de estos medicamentos ${ }^{20}$.

Directamente ligada a las características administrativas de las clínicas está la prevalencia de las enfermedades. Mientras que en el HUSVF todos los pacientes, independientemente de su enfermedad, al estar anticoagulados pueden acudir a la clínica de anticoagulación para su control, en el HUFSFB esto no es de carácter obligatorio y los médicos tratantes deciden o no acudir a los servicios de la clínica para el control de sus pacientes, lo cual reduce en gran medida la prevalencia de enfermedades crónicas como la fibrilación auricular. De igual manera esto está ligado al tratamiento farmacológico, ya que el uso de antagonistas de la vitamina $\mathrm{K}$ hace necesario el seguimiento periódico para el control del INR, por lo cual todos los pacientes del HUSVF están en permanente seguimiento por la clínica, y por el contrario, en el HUFSFB, el uso de los ACOD reduce la necesidad de seguimiento periódico y facilita el manejo de los pacientes por sus médicos tratantes.

De acuerdo con lo referido en la literatura, casi el $50 \%$ de los eventos tromboembólicos provocados están asociados a una hospitalización reciente como factor desencadenante y alrededor del $30 \%$ se tratan de eventos no provocados ${ }^{16}$. En la actualidad se ha incrementado la frecuencia de eventos tromboembólicos no provocados, reportando una frecuencia de alrededor del $45-50 \%$ según el registro RIETE ${ }^{21,22}$. En el HUSVF se encontró que el $58 \%$ de los pacientes anticoagulados por enfermedad tromboembólica venosa era debido a eventos no provocados. En el HUFSFB, la mayoría de los eventos fueron provocados, entre los cuales los principales factores desencadenantes fueron la inmovilidad y el cáncer.

Al observar el seguimiento y el número de visitas, se encuentra que en el HUSVF el seguimiento fue mayor, al igual que el número de visitas, lo cual seguramente está en relación con la mayor prevalencia de fibrilación auricular, una enfermedad crónica que requiere anticoagulación indefinida ${ }^{18}$, mientras que en el HUFSFB el número de seguimientos y de visitas fue menor, posiblemente relacionado con que la enfermedad más prevalente fue la enfermedad tromboembólica venosa, que en muchos casos solo requiere anticoagulación por periodos que van de 3 a 6 meses $^{19}$.

En los pacientes anticoagulados con warfarina, los valores altos de TRT se han correlacionado con una reducción de las complicaciones como la trombosis y el sangrado ${ }^{21}$. Las guías de práctica clínica, especialmente para fibrilación auricular, recomiendan que el 
control óptimo del TRT en los pacientes se encuentre por encima del $70 \%{ }^{18,23}$. Sin embargo, en la práctica clínica, el TRT obtenido usualmente es más bajo al compararlo con el de los estudios clínicos aleatorizados y cuando se comparan las clínicas de anticoagulación con la práctica comunitaria ${ }^{24}$.

Miranda, et al. ${ }^{24}$ reportaron un TRT del $54 \%$ en la clínica de anticoagulación del HUSVF para el periodo comprendido entre 2011 y 2012. En este estudio se observa que el TRT se conserva respecto al obtenido en el anterior estudio (55 vs $54 \%$ ), lo cual está relacionado con los cambios realizados en la calidad de la atención, el registro de los datos y la unificación de la información. Sin embargo, se deben realizar esfuerzos por desarrollar estrategias con el fin de elevar el TRT hasta lo recomendado por las guías internacionales ${ }^{25,26}$. Como limitantes, en esta institución se encuentra que alrededor del $90 \%$ de los pacientes aún se encuentran con warfarina genérica, los pacientes en ocasiones pierden el acceso a la continuidad de la clínica y algunos INR se determinan fuera del laboratorio institucional.

El HUFSFB registraba en el pasado estudio un TRT del 53\%, el cual aumentó en 2 años hasta el $69.6 \%$, acercándose a los estándares internacionales; este aumento se debe probablemente a las mejoras realizadas en la clínica, que incluyeron ajustes por vía telefónica y virtual, y mejoras en la educación de los pacientes $^{12,13}$. Vale la pena mencionar que, desafortunadamente, un porcentaje importante de los pacientes anticoagulados con warfarina tuvieron que ser excluidos del estudio, ya que el registro de los INR ajustados telefónicamente no estaba incluido en la historia clínica. Aquí vemos una oportunidad de mejoramiento en el manejo de la clínica para fortalecer el seguimiento y evitar el subregistro de la información.

El sangrado es la principal complicación de la terapia anticoagulante ${ }^{27}$. El riesgo de sangrado mayor con antagonistas de la vitamina $\mathrm{K}$ es dependiente de la calidad del control de la anticoagulación, con una tasa que puede llegar a 1,3/100 pacientes por año en aquellos con un INR entre 2 y $3^{28,29}$. Por el contrario, los ACOD han demostrado un mejor perfil de seguridad en comparación con la warfarina; en el caso de la fibrilación auricular, un metaanálisis de los principales estudios de fase III encontró una hazard ratio general de 0.48 para sangrado mayor intracraneano ${ }^{30}$.

En este estudio, con respecto al sangrado, se encontraron grandes diferencias entre las dos instituciones. En el HUSVF, el sangrado total fue tres veces mayor que en el HUFSFB, lo cual tiene una relación directa con el tratamiento anticoagulante utilizado y el TRT.
Cuando vemos los resultados, observamos que en la población del HUSVF el sangrado mayor fue del $1.88 \%$, lo cual está acorde con los reportes de la literatura sobre sangrado mayor con el uso de antagonistas de la vitamina $\mathrm{K}$, mientras que en el HUFSFB, donde el sangrado mayor fue del $0.55 \%$, está en relación con el mayor uso de ACOD y coincide con los reportes de sangrado mayor de estos medicamentos ${ }^{31,32}$.

Al comparar en el tiempo el caso del HUFSFB es interesante observar cómo se han mejorado los desenlaces tanto en los pacientes con antagonistas de la vitamina $\mathrm{K}$ como en aquellos con ACOD; probablemente se deba a la implementación de diferentes estrategias educativas audiovisuales, charlas y asesoramiento telefónico que se han venido realizando con el fin de reforzar la adherencia y el correcto uso de los medicamentos, sumado a la realización de los controles ambulatorios. Para futuros estudios sería primordial evaluar la adherencia de los pacientes a los ACOD en el tiempo, dado al aumento del uso de estos medicamentos en el tratamiento anticoagulante, y así poder realizar un análisis de las implicaciones en los desenlaces.

Como limitantes de este estudio está la naturaleza retrospectiva de su diseño, lo que hace que exista algún sesgo en la suficiencia de la información. En las dos instituciones se detectaron problemas en el registro de algunos datos, como la estandarización del registro del sangrado en la historia clínica de seguimiento en el HUSVF y el registro de todos los INR que se reportan en consultas telefónicas en el HUFSFB.

\section{Conclusiones}

Las diferencias administrativas entre las dos instituciones se ven reflejadas en los resultados obtenidos. Existe, ligada al sistema de salud de nuestro país, una gran diferencia en la población, las enfermedades y el acceso a los servicios y a los medicamentos, lo cual tiene repercusiones directas en el desenlace de los pacientes, en especial en términos de seguridad. Es muy importante fortalecer la creación de clínicas de anticoagulación en el país con el fin de hacer un seguimiento estricto de este grupo de pacientes, que son de alta complejidad. En las clínicas ya creadas se hace necesario buscar estrategias para mejorar el registro de los datos y continuar optimizando los indicadores de calidad para que esto se vea reflejado en el bienestar de los pacientes. Se deben encaminar también esfuerzos para que los ACOD sean incluidos en el plan obligatorio de salud en estas indicaciones, con el fin de generar a todos los pacientes los beneficios 
ampliamente reportados y evidenciados en nuestra población, en especial en la reducción del riesgo de sangrado. Hace falta aún investigación en estas aéreas para caracterizar mejor nuestra población y poder realizar guías enfocadas hacia los pacientes colombianos.

\section{Financiamiento}

El estudio fue financiado con recursos propios y con el apoyo de ambas instituciones de salud.

\section{Conflicto de intereses}

Los autores declaran que no existe conflicto de intereses.

\section{Responsabilidades éticas}

Protección de personas y animales. Los autores declaran que para esta investigación no se han realizado experimentos en seres humanos ni en animales.

Confidencialidad de los datos. Los autores declaran que han seguido los protocolos de su centro de trabajo sobre la publicación de datos de pacientes.

Derecho a la privacidad y consentimiento informado. Los autores declaran que en este artículo no aparecen datos de pacientes.

\section{Bibliografía}

1. Holbrook A, Schulman S, Witt DM, Vandvik PO, Fish J, Kovacs MJ, et al. Evidence-based management of anticoagulant therapy: Antithrombotic Therapy and Prevention of Thrombosis, $9^{\text {th }}$ ed: American College of Chest Physicians Evidence-Based Clinical Practice Guidelines. Chest. 2012;141(2 Suppl): e152S-84S.

2. Lip GY. Stroke prevention in atrial fibrillation. Eur Heart J. 2017;38:4-5.

3. Lip GY, Lim HS. Atrial fibrillation and stroke prevention. Lancet Neurol. 2007;6:981-93.

4. Ageno W, Gallus AS, Wittkowsky A, Crowther M, Hylek EM, Palareti G, et al. Oral anticoagulant therapy: Antithrombotic Therapy and Prevention of Thrombosis, $9^{\text {th }}$ ed: American College of Chest Physicians Evidence-Based Clinical Practice Guidelines. Chest. 2012;141(2 Suppl): e44S-88S.

5. García DA, Baglin TP, Weitz JI, Samama MM; Physicians ACoC. Parenteral anticoagulants: Antithrombotic Therapy and Prevention of Thrombosis, $9^{\text {th }}$ ed: American College of Chest Physicians Evidence-Based Clinical Practice Guidelines. Chest. 2012;141(2 Suppl): e24S-43S.

6. Fuster V, Rydèn LE, Cannom DS, Crijns HJ, Curtis AB, Ellenbogen KA, et al. 2011 ACCF/AHA/HRS focused updates incorporated into the ACC/ AHA/ESC 2006 Guidelines for the management of patients with atrial fibrillation: a report of the American College of Cardiology Foundation/ American Heart Association Task Force on Practice Guidelines developed in partnership with the European Society of Cardiology and in collaboration with the European Heart Rhythm Association and the Heart Rhythm Society. J Am Coll Cardiol. 2011;57:e101-9.

7. Franchini M, Bonfanti C, Mannucci PM. Management of bleeding associated with new oral anticoagulants. Semin Thromb Hemost. 2015;41: 788-801.

8. Barnes GD, Froehlich JB. Anticoagulation: where we are and where we need to go. J Thromb Thrombolysis. 2009;28:220-3.

9 Ryan F, Byrne S, O'Shea S. Managing oral anticoagulation therapy: improving clinical outcomes. A review. J Clin Pharm Ther. 2008;33:581-90.
10. Huisman MV, Rothman KJ, Paquette M, Teutsch C, Diener HC, Dubner SJ, et al. The changing landscape for stroke prevention in AF: findings from the GLORIA-AF Registry Phase 2. J Am Coll Cardiol. 2017;69:777-85.

11. Ocampo C, Hernández $O$, Velásquez C, Tobón I, Mejía F. La clínica de anticoagulación del Hospital Universitario San Vicente de Paúl: demografía, efectividad y complicaciones. IATREIA 2004;17:105-14.

12. Taboada $L$, Silva $L$, Montenegro A. Beneficios de la clínica de anticoagulación. Act Med Colomb. 2013;38:239.

13. Laverde LP, Gómez SE, Montenegro AC. Experiencia de una clínica de anticoagulación. Rev Colomb Cardiol. 2015;22:224-30.

14. Heit J. Epidemiology of venous thromboembolism. Nature Rev Cardiol. 2015;12:464-74.

15. Ageno W, Squizzato A, Garcia D, Imberti D. Epidemiology and risk factors of venous thromboembolism. Semin Thromb Hemost. 2006;32:651-8.

16. Wendelboe A, Raskob G. Global burden of thrombosis. Circulation Res. 2016;118:1340-7.

17. Chugh S, Havmoeller R, Narayanan K, Singh D, Rienstra M, Benjamin E, et al. Worldwide epidemiology of atrial fibrillation: a global burden of disease 2010 study. Circulation. 2013;129:837-47.

18. Kirchhoff $P$, Benussi S, Kotecha D, Ahlsson A, Atar D, Casadei B, et al. 2016 ESC Guidelines for the management of atrial fibrillation developed in collaboration with EACTS. Eur Heart J. 2016;37:2893-962.

19. Kearon C, Akl E, Ornelas J, Blaivas A, Jiménez D, Bounameaux $H$, et al. Antithrombotic therapy for VTE disease. Chest. 2016;149:315-52.

20. Camm A, Accetta G, Ambrosio G, Atar D, Bassand J, Berge E, et al. Evolving antithrombotic treatment patterns for patients with newly diagnosed atrial fibrillation. Heart. 2016;103:307-14.

21. Spencer FA, Gore JM, Reed G, Lessard D, Pacifico L, Emery C, et al. Venous thromboembolism and bleeding in a community setting. The Worcester Venous Thromboembolism Study. Thromb Haemost. 2009;101:878-85.

22. Tzoran I, Brenner B, Papadakis M, Di Micco P, Monreal M. VTE Registry: what can be learned from RIETE? Rambam Maimonides Med J. 2014:5:e0037.

21. Connolly SJ, Pogue J, Eikelboom J, Flaker G, Commerford P, Franzosi MG, et al. Benefit of oral anticoagulant over antiplatelet therapy in atrial fibrillation depends on the quality of international normalized ratio control achieved by centers and countries as measured by time in therapeutic range. Circulation. 2008;118:2029-37.

22. Cortelazzo S, Finazzi G, Viero P, Galli M, Remuzzi A, Parenzan L, et al. Thrombotic and hemorrhagic complications in patients with mechanical heart valve prosthesis attending anticoagulation clinic. Thromb Haemost. 1993;69:316-20.

23. Baker WL, Cios DA, Sander SD, Coleman Cl. Meta-analysis to assess the quality of warfarin control in atrial fibrillation patients in the United States. J Manag Care Pharm. 2009;15:244-52.

24. Miranda H, Osorio S, Giraldo DP, Duque J, Cataño JU, Tobón LI, et al. Tiempo en rango terapéutico en clínica de anticoagulación, reportes de eventos adversos y factores asociados a bajo TRT. Acta Med Colomb. 2016;41:42-8.

25. Connolly SJ, Pogue J, Eikelboom J, Flaker G, Commerford P Franzosi MG, et al. Depends on the quality of international normalized ratio control achieved by benefit of oral anticoagulant over antiplatelet therapy in atrial fibrillation centers and countries as measured by time in therapeutic range. Circulation. 2008;118:2029-37.

26. Lader E, Martin N, Cohen G, Meyer M, Reiter P, Dimova A, et al. Warfarin therapeutic monitoring: is $70 \%$ time in the therapeutic range the best we can do? J Clin Pharm Ther. 2012;37:375-7.

27. Niessner A, Tamargo J, Morais J, Koller L, Wassmann S, Husted S, et al. Reversal strategies for non-vitamin $\mathrm{K}$ antagonist oral anticoagulants: a critical appraisal of available evidence and recommendations for clinical management - a joint position paper of the European Society of Cardiology Working Group on Cardiovascular Pharmacotherapy and European Society of Cardiology Working Group on Thrombosis. Eur Heart J. 2017;38:1710-6.

28. Gallego P, Roldán V, Marín F, Romera M, Valdés M, Vicente V, et al. Cessation of oral anticoagulation in relation to mortality and the risk of thrombotic events in patients with atrial fibrillation. Thromb Haemost. 2013; 110:1189-1198.

29. De Caterina R, Husted S, Wallentin L, Agnelli G, Bachmann F, Baigent C, et al. Anticoagulants in heart disease: current status and perspectives. Eur Heart J. 2007;28:880- 913.

30. Ruff CT, Giugliano RP, Braunwald E, Hoffman EB, Deenadayalu N, Ezekowitz MD, et al. Comparison of the efficacy and safety of new oral anticoagulants with warfarin in patients with atrial fibrillation: a meta-analysis of randomised trials. Lancet. 2014;383:955-62.

31. Eikel J, Merli G. Bleeding with direct oral anticoagulants vs warfarin: clinical experience. Am J Med. 2016;129:S33-S40.

32. Adeboyeje G, Sylwestrzak G, Barron JJ, White J, Rosenberg A, Abarca J, et al. Major bleeding risk during anticoagulation with warfarin, dabigatran, apixaban, or rivaroxaban in patients with nonvalvular atrial fibrillation. J Manag Care Spec Pharm. 2017;23:968-78. 